Article published in JCP 149, 214502 (2018)

\title{
Inflection point in the Debye relaxation time of 2-butyl-1-octanol
}

\author{
Erik Thoms, ${ }^{1,2, a)}$ Sławomir Kołodziej, ${ }^{1,2}$ Michał Wikarek, ${ }^{1,2}$ Stefan Klotz, ${ }^{3}$ Sebastian Pawlus, ${ }^{1,2}$ and Marian \\ Paluch ${ }^{1,2, b)}$ \\ 1) Institute of Physics, University of Silesia, 75 Putku Piechoty 1, 41-500 Chorzów, \\ Poland \\ ${ }^{2)}$ Silesian Center for Education and Interdisciplinary Research, 75 Putku Piechoty 1A, 41-500 Chorzów, \\ Poland. \\ 3) IMPMC, UMR 7590, Sorbonne Université, 4 Place Jussieu, 75252 Paris, France
}

(Dated: 21 October 2019)

We report a striking anomaly in the pressure dependent Debye-relaxation time of the branched monohydroxy alcohol 2-butyl-1-octanol. Evidence of a crossover from slower to faster than exponential pressure dependency was obtained at different temperatures via high pressure broadband dielectric spectroscopy. At the same time, viscosity measurements reveal similar behavior in the viscosity respectively the structural relaxation time, indicating a similar origin of the phenomena.

\section{INTRODUCTION}

The study of monohydroxy alcohols (MAs) is an effective way to examine the influence of hydrogen bonds on the molecular dynamics. Like other representatives of this class of materials, the branched monohydroxy alcohol 2-butyl-1-octanol (2B1O, $\left.\mathrm{C}_{12} \mathrm{H}_{25} \mathrm{OH}\right)$ tends to supercool and eventually form a glass upon isobaric cooling or isothermal pressurization. Whilst many glass formers share similar molecular dynamics in the supercooled state - as evidenced, e.g., by analogous features in dielectric relaxation spectra - monohydroxy alcohols, as well as some other H-bonded materials ${ }^{1}$, exhibit an additional relaxation at low frequencies. A thorough review on this phenomenon is given by Böhmer et al. ${ }^{2}$. Interestingly, the associated peak in the dielectric loss spectrum $\epsilon^{\prime \prime}(f)$ with peak frequency $f_{\max }$ has been reported to follow a Debye behavior, and can thus be well described by the Debye equation

$$
\epsilon^{\prime \prime}=\frac{\Delta \epsilon_{D} \times \omega \tau_{D}}{1+\omega^{2} \tau_{D}^{2}}
$$

where $\Delta \epsilon_{D}$ is the relaxation strength, $\omega=2 \pi f$ the angular frequency, and $\tau_{D}$ the relaxation time. However, it should be mentioned that some recent reports indicate a slight broadening of the relaxation ${ }^{3,4}$, and promote fitting with either the empirical Havriliak-Negami (HN) equation $^{5}$

$$
\epsilon^{\prime \prime}=\frac{\Delta \epsilon_{D} \times \sin \left(\beta \times \arctan \left(\frac{\left(\omega \tau_{D}\right)^{\alpha} \sin (\pi \alpha / 2)}{\left(1+\left(\omega \tau_{D}\right)^{\alpha} \cos (\pi \alpha / 2)\right.}\right)\right)}{\left(1+2\left(\omega \tau_{D}\right)^{\alpha} \cos (\pi \alpha / 2)+\left(\omega \tau_{D}\right)^{2 \alpha}\right)^{\beta / 2}}
$$

(where $\alpha$ and $\beta$ are the symmetric and the asymmetric broadening parameters, respectively), or with the

\footnotetext{
a) erik.thoms@smcebi.edu.pl

b) marian.paluch@us.edu.pl
}

closely related Cole-Davidson (CD) equation ${ }^{6}$ (Eq. 2 with $\alpha=1$ ). The broadening, common to the canonical structural relaxation peaks, is usually associated with a distribution of relaxation times due to different energetic environments for molecules in real materials, respectively with an increase in cooperativity in the dynamics.

For an explanation of the peculiar Debye-like relaxation, it is required to consider the formation of supermolecular structures often found in H-bonded liquids. In particular, (stretched) circular or linear structures formed by the hydroxyl groups have been found in MAs by molecular dynamics simulations ${ }^{7,8}$ and experiment $^{9,10}$. The later structures are generally assumed to be the cause of the Debye-like relaxations ${ }^{2,11}$, with the transient chain model proposed by Gainaru et al. $^{10}$ gaining popularity ${ }^{12,13}$. As a result, preventing the formation of $\mathrm{H}$-bonds either via chemical alteration or application of pressure will cause the Debye-peak in the dielectric loss spectrum to vanish ${ }^{14,15}$. Likewise, a promotion of ring-like superstructures over linear ones alters the dielectric response, again decreasing the strength of the Debye-like relaxation. In this paper we present the results of broadband dielectric spectroscopy measurements on 2-butyl-1-octanol at ambient and elevated pressure up to $1.8 \mathrm{GPa}$, focussing on the Debye-like relaxation. Analyzing the pressure dependence of $\tau_{D}$, we find a crossover from slower to faster than exponential behavior, a feature reported before only for the structural relaxation, as well as the related properties of viscosity $\eta$ and dcconductivity $\sigma_{d c}$. To confirm the presence of this phenomenon in $\eta$, viscosity measurements up to $1.2 \mathrm{GPa}$ were performed at room temperature.

\section{EXPERIMENTAL DETAILS}

2-butyl-1-octanol was purchased from Simga-Aldrich and used as received. The dielectric measurements were performed using a Novocontrol Alpha-A impedance analyzer. The pressure was applied in a high pressure cham- 
ber via silicon oil. The sample fluid was only in contact with the stainless steel capacitor and Teflon. For temperature control, the pressure chamber was placed in a Tenney Junior compact temperature test chamber with an uncertainty of $0.5 \mathrm{~K}$. The viscosity measurements were performed using the rolling ball method ${ }^{16}$ within a sapphire cell with half-inch sapphire sphere anvils with a $3.5 \mathrm{~mm}$ flat culet. The CuBe gaskets were provided with a $1.3 \mathrm{~mm}$ hole with $0.5 \mathrm{~mm}$ depth, in which the speed of a tungsten carbide ball of typically $100 \mu \mathrm{m}$ diameter was observed at various inclination angles between $10^{\circ}$ and $20^{\circ}$. The force of up to 5 tons was provided by a VX2 Paris-Edinburgh cell ${ }^{17}$. Pressures were measured using the fluorescence of a BaFCl:Sm${ }^{2+}$ crystal inside the pressure chamber using a pressure coefficient of $+1.1 \mathrm{~nm} / \mathrm{GPa}^{18}$.

\section{RESULTS}

In Fig. 1 (A), selected ambient pressure dielectric loss spectra are shown for four temperatures, together with four curves collected at $T=198 \mathrm{~K}$ at different pressures. The most prominent feature for all curves is the Debye-like peak at low frequencies, e.g., for the $178 \mathrm{~K}$ curve at $1 \mathrm{~Hz}$. The structural relaxation (often named $\alpha$-relaxation) can be observed as a shoulder in the high frequency flank of the Debye-like peak. An additional contribution at the highest frequencies indicates the secondary, or $\beta$-, relaxation. Since all of the above features shift towards higher frequencies with rising temperature, marking a decrease in their respective relaxation times, the secondary relaxation peak is beyond the range of the spectrum at the higher two temperatures. In the structural relaxation, the shift can be observed from $1 \mathrm{kHz}$ at $178 \mathrm{~K}$ to $100 \mathrm{kHz}$ at $199 \mathrm{~K}$ and in the Debye-like relaxation from $1 \mathrm{~Hz}$ to $300 \mathrm{~Hz}$ over the same temperature range. It also becomes obvious that the intensity of the Debye relaxation decreases to less than half over this temperature range, from $\epsilon_{\max }^{\prime \prime}=1.2$ to 0.5 . This corresponds well with the data presented by Gao et al. earlier ${ }^{3}$.

Considering the curves collected isothermally at $T=$ $198 \mathrm{~K}$ and elevated pressures, the same features are present. The pressures are selected to achieve isochronal conditions, i.e., the respective Debye relaxation times match with the ambient pressure curves. This allows direct comparison of the Debye peaks under different thermodynamic conditions. Contrasted with the wellstudied type I monoalcohol 2-ethyl-1-hexanol ${ }^{19}$, it can be noticed that 2-butyl-1-octanol is more sensitive to pressure change, as evidenced by the comparatively stronger frequency shift of the Debye-like peak upon compression. Analogous to cooling, pressurization of the sample leads to an increase in the intensity of the dominant relaxation. It can clearly be seen, however, that relative to the associated isochronal ambient pressure curves, the intensity of the relaxation is higher for spectra obtained at elevated pressures. Such behavior is typical for type

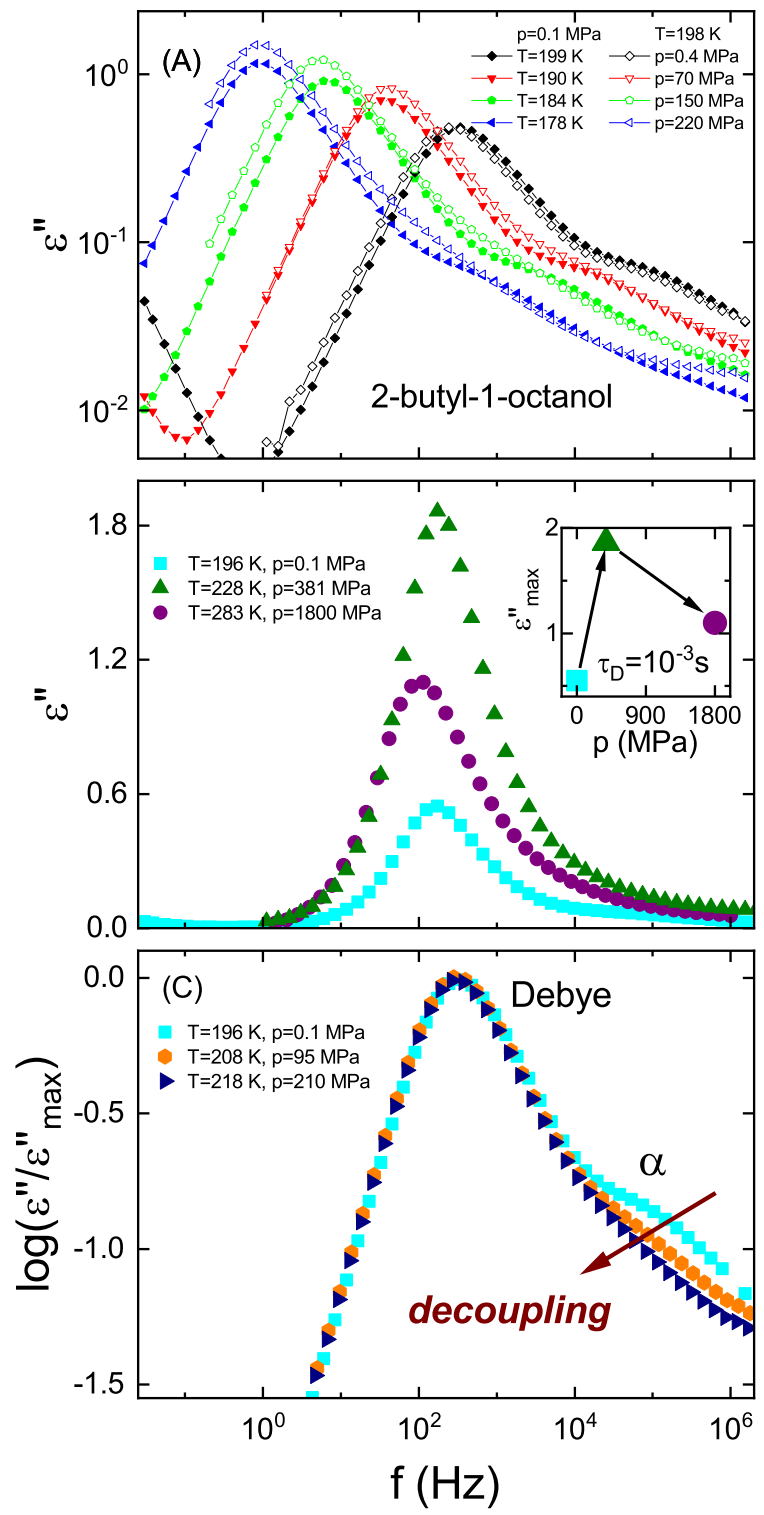

FIG. 1. (A) Frequency dependent dielectric loss of 2-butyl-1octanol at ambient pressure for selected temperatures (open symbols), together with data taken at $198 \mathrm{~K}$ with increased $p$ to match $\tau_{D}$ (closed symbols). The lines serve to guide the eyes. (B) The Debye-peak at isochronal conditions for three spectra with rising $p$ and $T$. The inset gives the change of peak height with increasing pressure. (C) Debye-peak at isochronal conditions for three spectra normalized by peak height. The arrow indicates a shift of the $\alpha$ - towards the Debye-relaxation peak with increasing $p$ and $T$.

II monoalcohols with the hydroxyl group located at a non-terminal position, which tend to form ring-like superstructures due to the caused steric shielding ${ }^{9,12,20}$. Increasing pressure can break these rings and promote open chain-like structures instead, thus increasing the dielectric response of the system. It should be noted, however, 
that the increase observed in 2-butyl-1-octanol is comparatively weaker than in typical type II monoalcohols. For example, the height of the Debye-like peak $\epsilon_{\max }^{\prime \prime}$ increases by a factor of approximately 2.5 from $p=0.4$ to $150 \mathrm{MPa}$, as opposed to a factor of $>4$ in 5-methyl-3heptanol at a similar pressure range and temperature ${ }^{21}$.

Interestingly, the amplitude does not change monotonically. This can be seen by the three isochronal spectra $\left(\tau_{D} \approx 10^{-3} \mathrm{~s}\right)$ presented in Fig. 1(B). $\epsilon_{\max }^{\prime \prime}$ more then triples from 0.55 at ambient pressure to 1.86 at $380 \mathrm{MPa}$, before decreasing again to 1.10 at $1.8 \mathrm{GPa}$, approximately twice the ambient pressure value. These opposing trends indicate that compression has at least two differing effects on the superstructure: At low $p$, the promotion of chain-like over ring-like formations is prevalent, increasing the number of transient chains and thus, the intensity of the relaxation, as reported for several type II MAs ${ }^{12,21}$. Above a threshold pressure, there is no further increase in the amount of chains. Instead, $\epsilon_{\text {max }}^{\prime \prime}$ is reduced. Such a decrease has been reported for type I alcohols before, and can be ascribed to the high packing density inhibiting the formation of long chains ${ }^{20}$. Wikarek et al. found similar non-monotonic behavior in 2-methyl-2-hexanol ${ }^{12}$.

Both the relaxation times of $\alpha$ - and Debye-relaxation increase upon pressurization or cooling, causing the relaxation peaks in Fig. 1 (A) to shift towards lower frequencies. However, they do not move in unison, as pointed out in Fig. 1 (C). Here, the loss for three isochronal spectra at different thermodynamic conditions is normalized by $\epsilon_{\max }^{\prime \prime}$. Consequently, the Debye-like peaks exhibit nearly perfect overlap. The $\alpha$-peak, on the other hand, shifts visibly towards the dominant feature upon an increase in $p$ and $T$ : at ambient pressure, the $\alpha$-relaxation is clearly visible as a shoulder at the high frequency flank of the Debye-like peak at $f \approx 10^{5} \mathrm{~Hz}$, while at $p=210 \mathrm{MPa}$, it appears merely as an excess contribution above $10^{4} \mathrm{~Hz}$. The change in the relative peak positions implies a decoupling between $\alpha$ - and Debye relaxation time.

To obtain $\tau_{D}$ and $\Delta \epsilon_{D}$, the experimental data has been fitted. It is known for other MAs that pressurization decreases the time separation between $\alpha$ - and Debye-like relaxation ${ }^{19,22}$, making the determination of $\tau_{\alpha}$ at elevated pressure challenging at least. Therefore, special care must be taken upon the selection of the model. Different fits for one exemplary ambient pressure spectrum at $T=181 \mathrm{~K}$ are shown in Fig. 2. First, a superposition of the CD- and the HN-model for the dominant and structural relaxation, respectively, is given by the continuous lines. The dashed lines show an alternative fit, employing the Debye- and HN-model instead. To account for the difference between relaxation frequency and the frequency of the peak maximum at asymmetric broadened relaxations (i.e., $\beta \neq 1$ ), the mean relaxation time was obtained. It can be seen that both $\tau_{\alpha}$ and $\Delta \epsilon_{\alpha}$ vary strongly between the two fits, with the relaxation time being nearly one decade higher in the case of Debye and $\mathrm{HN}\left(\tau_{\alpha} \approx 10^{-3} \mathrm{~s}\right)$ than for $\mathrm{CD}$ and $\mathrm{HN}\left(1.3 \times 10^{-4} \mathrm{~s}\right)$.

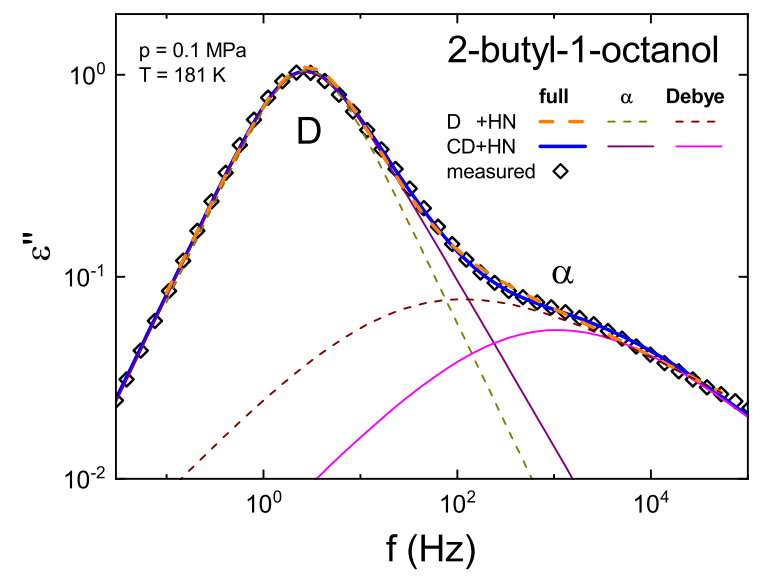

FIG. 2. Exemplary ambient pressure spectrum (diamonds). The dashed lines represent the best fit with one Debye- and one HN-relaxation (Eq. 1 and 2, respectively), the continuous lines the best fit with one CD- and one HN-relaxation. The components for Debye-like and $\alpha$-relaxation are shown separately.

On the other hand, in accord with the obtained fit parameters, the Debye peak position is largely independent from the model used $\left(\tau_{D}=5.7 \times 10^{-2}\right.$ and $4.8 \times 10^{-2} \mathrm{~s}$, respectively). The best results were obtained with the combination of $\mathrm{CD}$ and $\mathrm{HN}$, which therefore was used for all subsequent fits.

For a thorough examination of the $p$-dependency of the Debye process, spectra at elevated pressures and eight temperatures between $T=198$ and $263 \mathrm{~K}$ were acquired and fitted as described above. The resulting $\tau_{D}(p)$ isotherms are presented in Fig. 3(A). For all temperatures, $\tau_{D}$ increases with pressure, with a higher slope at lower $T$. At low temperatures, it can be seen that the curves are convex against the x-axis, i.e., the increase is super-Arrhenius like and can be described by the pressure equivalent ${ }^{23}$ of the Vogel-Fulcher-TammanHesse (VF) equation:

$$
\tau_{D}(p)=\tau_{D, 0} \times \exp \left(\frac{C_{F} p}{p_{\infty}-p}\right)
$$

Here, $\tau_{D, 0}=\tau_{D}(p \rightarrow 0)$ can be approximated by the value at ambient pressure. $p_{\infty}$ indicates a divergence point, while the strength parameter $C_{F}$ quantifies the discrepancy from ideal volume activated behavior given by $\tau=\tau_{0} \times \exp (p \Delta V / R T)$, the pressure equivalent of the Arrhenius equation. At higher $T, \mathrm{VF}$-behavior can still be observed for increased pressures, e.g., for the $218 \mathrm{~K}$ isotherm above $p=250 \mathrm{MPa}$. This conforms well with previous results on MAs, reporting either Arrheniuslike ${ }^{12,24}$ or super-Arrhenius-like $\mathrm{e}^{22}$ pressure dependence of the Debye relaxation time. Close inspection, however, reveals that $\tau_{D}(p)$ deviates from these behaviors at low pressures. This effect is more obvious at higher tem- 


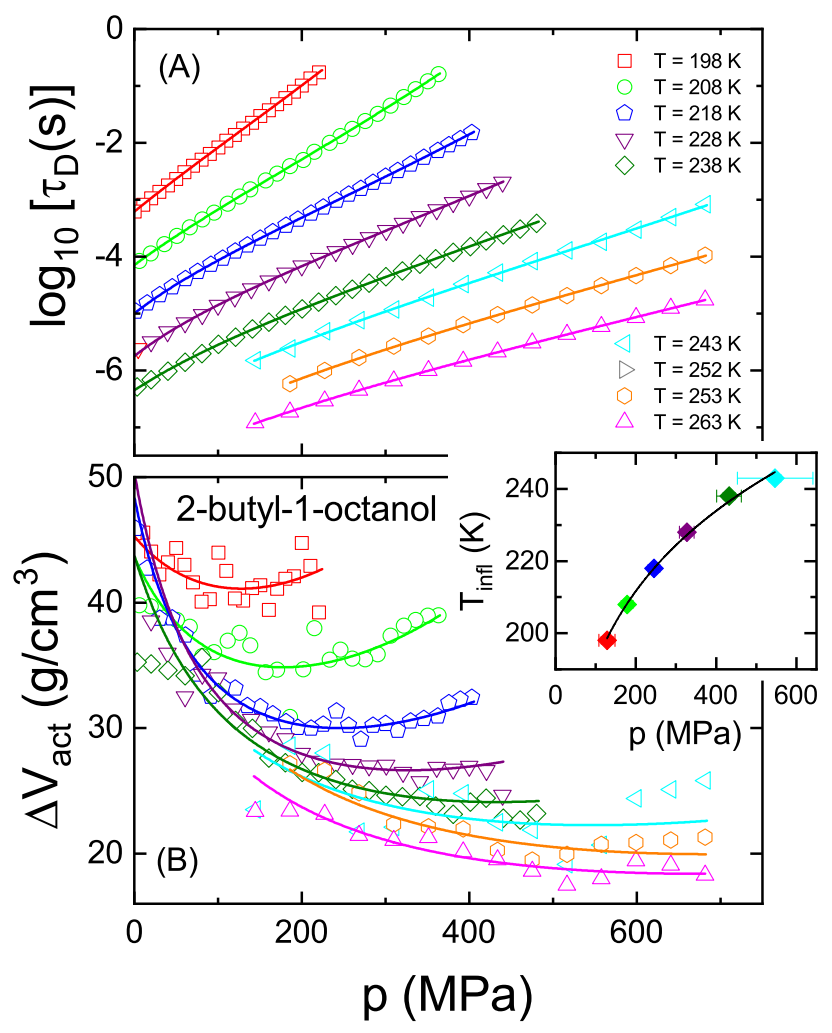

FIG. 3. Pressure dependency of the Debye relaxation time (A) and according activation volume (B) of 2-butyl-1-octanol for six isotherms. The lines represent fits with the hybrid model (Eq. 5). The inset shows the $p$-dependence of the inflection temperature, with the bars marking the maximum error, and the line an Andersson-Andersson fit.

peratures. For instance, the $238 \mathrm{~K}$-isotherm can clearly be seen to have a concave shape against the $p$-axis below $200 \mathrm{MPa}$, indicating slower than exponential pressure dependency.

It is convenient to study this phenomenon in terms of the activation volume:

$$
\Delta V_{a c t}(p)=R T \times\left(\frac{\partial \ln \left(\tau_{D}\right)}{\partial p}\right)
$$

with the gas constant $R . \Delta V_{a c t}(p)$ is presented in Fig. $3(\mathrm{~B})$. In this presentation, slower than exponential pressure dependence of the relaxation time is given by a negative, faster than exponential (super-Arrhenius-like) behavior by a positive slope of $V_{a c t}(p)$. Clearly, the activation volume is decreasing upon compression for all isotherms at low pressures. Above a temperature dependent pressure, $V_{a c t}$ begins to increase. The resulting minimum $V_{a c t, \text { min }}$ marks the inflection pressure $p_{\text {infl }}$, i.e. the crossover point between the slower and faster than exponential ranges. Increasing the temperature, $V_{a c t, m i n}$ is observed at higher pressures. A more detailed analysis is possible using a phenomenological model proposed by Bair $^{25}$. Originally employed to describe inflections in $\eta(p)$, it combines the pressure equivalent of the VF equa- tion (Eq. 3) with the McEwen equation, to be applicable for high and low pressure ranges, respectively:

$$
\tau_{D}(p)=\tau_{D, 0} \times\left(1+\frac{\alpha_{0}}{q} p\right)^{q} \times \exp \left(\frac{C_{F} p}{p_{\infty}-p}\right)
$$

Supplementary to the factors taken from Eq. 3, $\alpha_{0}$ and $q$ are the McEwen parameter and exponent, respectively. Fits according to this hybrid model are shown in Fig. $3(\mathrm{~A})$, and the according activation volumes in (B). The inflection points, as obtained by the minima in the latter curves, are given in the inset in a $T_{\text {infl }}(p)$ presentation. For the highest two temperatures, the inflection points predicted by that method were located above the examined pressure range, and therefore are not shown. It can be seen that the inflection temperature increases with pressure. Closer inspection reveals that the increase is strong for low values of $p$, but becomes slower at rising pressures.

To describe the pressure dependence of the glass transition temperature, often a model originally proposed by Andersson and Andersson ${ }^{26}$ for Poly(propylene glycol) is employed:

$$
T_{g}=k_{1} \times\left(1+\frac{k_{2}}{k_{3}} p\right)^{1 / k_{2}}
$$

$k_{1}, k_{2}$ and $k_{3}$ are material constants. The equation is empirical, but can be linked ${ }^{27}$ to the entropy based Avramov model $^{28}$. It should be noted that the Simon equation, used to describe the melting point $T_{m}(p)$ of simple crystals, can be brought to the same form ${ }^{29,30}$. Interestingly, it also delivers a reasonable description of $T_{\text {infl }}(p)$ for 2B1O, as shown by the black line in the inset of Fig. 3, predicting $T_{\text {infl }}=26 \mathrm{~K}$ at very low pressures.

From Fig. 3 (A) it also becomes clear that the relaxation time, at which the inflection point can be found, decreases upon heating. It should be mentioned that this deviates from observations made in the $p$ dependence of the structural relaxation time of the molecular liquid propylene carbonate, where isochronal behavior of the inflection point has been reported ${ }^{31}$. Since the Debye-like relaxation is associated not with the structure of a single molecule, but with transient chain superstructures, it is reasonable to assume that the stability respectively the chain length influences the occurrence of the inflection. In the case of 2-butyl-1-octanol, it can be concluded that at higher pressures the average chain length at the crossover point is lower than at low $p$, thus decreasing the associated $\tau_{D}$ values.

This is the first time an inflection in the pressure dependent Debye relaxation time was reported. These results are of high interest, since similar phenomena have been observed before in various dynamic properties and different materials ${ }^{31-35}$. Additionally, Lederle et al. reported that an inflection in the temperature dependence of $\tau_{D}$ at ambient pressure seems to be common behavior in simple MAs, and explained it with the reduction of supermolecular chain length upon heating ${ }^{36}$. Of particular 


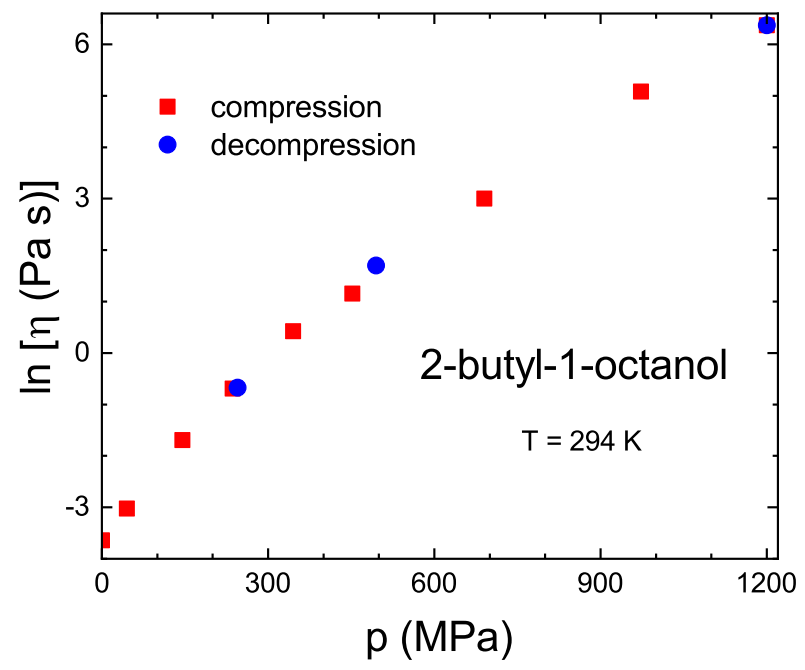

FIG. 4. Pressure-dependent viscosity of 2-butyl-1-octanol at $294 \mathrm{~K}$. Values taken at compression (red) and decompression (blue) show good agreement.

relevance is the finding of a change in the pressure dependence of viscosity $\eta$ at $\approx 0.5 \mathrm{GPa}$, and, with identical inflection pressures, $\tau_{\alpha}$ for two monohydroxy alcohols, 2-ethyl-1-hexanol and 5-methyl-2-hexanol by Pawlus et $a l .{ }^{37}$. It should be mentioned that for these materials, $p_{\text {infl }}$ also marks the threshold pressure below which thermodynamic scaling is valid ${ }^{37}$. As shown in Fig. 4, in the case of 2-butyl-1-octanol, $\eta(p)$ also shows a concave or slower than exponential development for an isotherm taken at $294 \mathrm{~K}$, and it can reasonably be assumed that the same is true for $\tau_{\alpha}(p)$. However, a change to convex behavior could not be observed in the examined pressure range, and thus must take place at a pressure higher than 1.2 GPa. Accordingly, a fit with only the McEwen equation (first term of a viscosity equivalent of Equ. 5) is sufficient to describe the data, with no apparent improvement when using the full hybrid equation (not shown). For comparison, extrapolating the Andersson-Andersson fit shown in the inset of Fig. 3 to $T=294 \mathrm{~K}$ yields $p_{\text {infl }} \approx 2 \mathrm{GPa}$.

The occurrence of the inflection has been attributed to the opposed pressure dependencies of the isothermal compressibility $\kappa_{T}$ and the apparent isochoric activation energy $E_{V}{ }^{33}$. Since $V_{\text {act }}$ is proportional to $\kappa_{T} \times E_{V}$, the minimum is caused by the crossover from the low pressure regime, where $\kappa_{T}$ is more sensitive to pressure than $E_{V}$, to the high pressure regime, where this relation is inverted. Note that this explanation was made to describe the viscosity $\eta$ of the canonical glass former propylene carbonate. For many simple materials, a coupling of structural relaxation time and $\eta$, as given by the Einstein-Debye relation, is expected. Therefore, a change from slower to faster than exponential behavior in $\eta$ would be reflected in $\tau_{\alpha}$. Pawlus et al. have show that for two monoalcohols this coupling, as well as thermody-

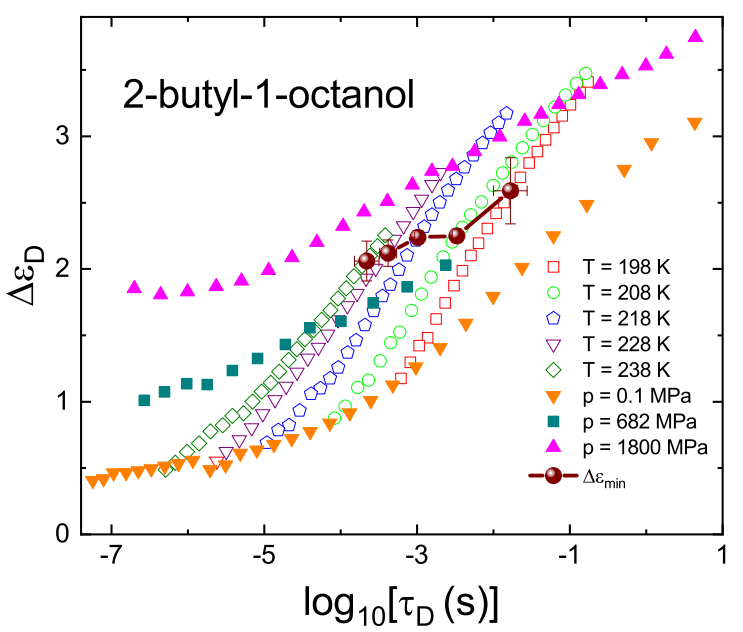

FIG. 5. Dielectric strength of the Debye process in dependence of its time constant for six isotherms (open symbols) and two isobars (closed symbols). The red spheres denote the respective inflection points.

namic scaling, only holds under a threshold pressure ${ }^{37,38}$. As shown in Fig. 1(C), however, the presence of a similar coupling between Debye and $\alpha$-relaxation upon pressurization can be excluded. In fact, ambient pressure data for $2 \mathrm{~B} 1 \mathrm{O}$ reported by Gao et $a .^{3}{ }^{3}$ also exhibits the absence of such a phenomenon. This is in accordance with multiple reports for numerous monoalcohols, describing a distinct change in $\tau_{D} / \tau_{\alpha}$ upon change of either temperature or pressure $20,21,36,39$. At the same time, the simultaneous presence of inflection points in both properties should not be ignored, as it hints towards a common, or at least similar, origin.

For a further examination of the inflection point and the Debye-like process, an overview over the dielectric strength $\Delta \epsilon_{D}$ in dependence of the according relaxation time $\tau_{D}$ for a selection of five isotherms and three isobars is given in Fig. 5. Additionally, for the five isotherms, the $\Delta \epsilon_{D}\left(\tau_{D}\right)$ values associated with the respective inflection points $p_{\text {infl }}$ are shown. The parameters were obtained using the fitting procedure described above. It can be noted that these values are located between the $682 \mathrm{MPa}$ and $1.8 \mathrm{GPa}$ isobars. In accordance with Fig. 1 , high relaxation times generally correspond to higher $\Delta \epsilon_{D}$. When examining the isobars, it becomes obvious that this increase of $\Delta \epsilon_{D}\left(\tau_{D}\right)$ takes part in two different regions: At low $\tau_{D}$, respectively high temperatures, a low slope is observed. Upon cooling, the slope increases markedly above a pressure- and temperature dependent critical relaxation time. At ambient pressure, this transition can be found at $\tau_{D} \approx 10^{-3.8} \mathrm{~s}$, while at $p=1.8 \mathrm{GPa}$ it occurs at $\approx 10^{-5.2} \mathrm{~s}$. The high temperature region can be associated with relatively weak hydrogen bonding, and thus, normal liquid behavior, while at lower $T$ the $\mathrm{H}$-bonds have distinct impact on the dynamic properties 
of the alcohol. For all temperatures, pressurisation yields a steep increase in $\Delta \epsilon_{D}\left(\tau_{D}\right)$, resulting in elevated dielectric strength at any given relaxation time when compared to the ambient pressure data. That indicates a breaking of H-bonded rings in favor of chain-like superstructures and is well consistent with the results presented in Fig. 1. At the highest relaxation times, i.e. highest pressures, a slight decrease in the slope can be noticed, indicating that further pressurisation antagonizes the generation of supermolecular chains, and decreases the amount or length of such structures.

\section{CONCLUSION}

Summarizing, we performed viscosity and dielectric spectroscopy measurements under elevated pressure on the monohydroxy alcohol 2-butyl-1-octanol. Focussing on the properties of the Debye-like relaxation found at lower frequencies than the structural $\alpha$-relaxation, we observe an increase in strength upon cooling or pressurisation. Similar to other type II MAs, we find that this effect is stronger for changes in $p$ than in $T$ when comparing isochronal Debye-like peaks, indicating the pressure induced transformation of ring-like to chainlike supermolecular structures. The effect, however, is non-monotonical, as at higher pressures the breaking of chain-like superstructures leads to a decrease of the relaxation strength. Most interestingly, examination of the Debye relaxation time shows behavior to our knowledge not reported before: upon pressurisation, a crossover from slower than exponential to faster than exponential (Vogel-Fulcher-like) progression is observed in $\log _{10}\left(\tau_{D}\right)(p)$. This is accompanied by slower than exponential pressure dependence of the viscosity. While a coupling of $\tau_{D}$ with $\eta$ respectively $\tau_{\alpha}$ is unlikely, the simultaneous occurrence of inflection points is remarkable and hints at a similar origin of these phenomena.

\section{ACKNOWLEDGMENTS}

S.P., M.P., S.K. and E.T. acknowledge the financial support of the project No. UMO-2015/17/B/ST3/01221 by the National Science Centre, Poland.

${ }^{1}$ K. Adrjanowicz, B. Jakobsen, T. Hecksher, K. Kaminski, M. Dulski, M. Paluch, and K. Niss, The Journal of Chemical Physics 143, 181102 (2015).

${ }^{2}$ R. Böhmer, C. Gainaru, and R. Richert, Physics Reports 545, 125 (2014).

${ }^{3}$ Y. Gao, W. Tu, Z. Chen, Y. Tian, R. Liu, and L.-M. Wang, The Journal of Chemical Physics 139, 164504 (2013).

${ }^{4} \mathrm{~S}$. Arrese-Igor, A. Alegría, and J. Colmenero, The Journal of Chemical Physics 146, 114502 (2017).

${ }^{5}$ S. Havriliak and S. Negami, J. Polym. Sci., Part C: Polym. Symp. 14, 99 (1966).

${ }^{6}$ D. W. Davidson and R. H. Cole, J. Chem. Phys. 18, 1417 (1950).
${ }^{7}$ J. L. MacCallum and D. P. Tieleman, Journal of the American Chemical Society 124, 15085 (2002).

${ }^{8}$ J. Lehtola, M. Hakala, and K. Hämäläinen, The Journal of Physical Chemistry B 114, 6426 (2010).

${ }^{9}$ W. Dannhauser, The Journal of Chemical Physics 48, 1911 (1968).

${ }^{10}$ C. Gainaru, R. Meier, S. Schildmann, C. Lederle, W. Hiller, E. A. Rössler, and R. Böhmer, Physical Review Letters 105 (2010), 10.1103/physrevlett.105.258303.

${ }^{11}$ P. Lunkenheimer, M. Michl, T. Bauer, and A. Loidl, The European Physical Journal Special Topics 226, 3157 (2017).

${ }^{12}$ M. Wikarek, S. Pawlus, S. N. Tripathy, A. Szulc, and M. Paluch, The Journal of Physical Chemistry B 120, 5744 (2016).

${ }^{13}$ I. Danilov, A. Pronin, E. Gromnitskaya, M. Kondrin, A. Lyapin, and V. Brazhkin, The Journal of Physical Chemistry B 121, 8203 (2017).

${ }^{14}$ O. E. Kalinovskaya, J. K. Vij, and G. P. Johari, The Journal of Physical Chemistry A 105, 5061 (2001).

${ }^{15}$ K. Grzybowska, S. Pawlus, M. Mierzwa, M. Paluch, and K. L. Ngai, The Journal of Chemical Physics 125, 144507 (2006).

${ }^{16}$ H. E. King, E. Herbolzheimer, and R. L. Cook, Journal of Applied Physics 71, 2071 (1992).

${ }^{17} \mathrm{~S}$. Klotz, G. Hamel, and J. Frelat, High Pressure Research 24, 219 (2004).

${ }^{18}$ Y. R. Shen, T. Gregorian, and W. B. Holzapfel, High Pressure Research 7, 73 (1991).

${ }^{19}$ D. Fragiadakis, C. M. Roland, and R. Casalini, The Journal of Chemical Physics 132, 144505 (2010).

${ }^{20}$ S. Pawlus, M. Wikarek, C. Gainaru, M. Paluch, and R. Böhmer, The Journal of Chemical Physics 139, 064501 (2013).

${ }^{21}$ C. Gainaru, M. Wikarek, S. Pawlus, M. Paluch, R. Figuli, M. Wilhelm, T. Hecksher, B. Jakobsen, J. C. Dyre, and R. Böhmer, Colloid and Polymer Science 292, 1913 (2014).

${ }^{22}$ S. Pawlus, M. Paluch, and M. Dzida, The Journal of Physical Chemistry Letters 1, 3249 (2010).

${ }^{23}$ M. Paluch, Z. Dendzik, and S. J. Rzoska, Physical Review B 60, 2979 (1999).

${ }^{24}$ G. P. Johari and W. Dannhauser, The Journal of Chemical Physics 50, 1862 (1969).

${ }^{25}$ S. Bair, High Temperatures - High Pressures 44, 415 (2015).

${ }^{26}$ S. P. Andersson and O. Andersson, Macromolecules 31, 2999 (1998).

${ }^{27}$ M. Paluch, S. Hensel-Bielowka, and T. Psurek, The Journal of Chemical Physics 113, 4374 (2000).

${ }^{28}$ I. Avramov, Journal of Non-Crystalline Solids 262, 258 (2000).

${ }^{29}$ F. Simon and G. Glatzel, Zeitschrift für anorganische und allgemeine Chemie 178, 309 (1929).

${ }^{30}$ N. H. March and M. P. Tosi, Introduction to Liquid State Physics (World Scientific Publishing, 2002).

${ }^{31}$ E. Thoms, Z. Wojnarowska, P. Goodrich, J. Jacquemin, and M. Paluch, The Journal of Chemical Physics 146, 181102 (2017), http://dx.doi.org/10.1063/1.4982941.

${ }^{32}$ R. L. Cook, H. E. King, C. A. Herbst, and D. R. Herschbach, The Journal of Chemical Physics 100, 5178 (1994).

${ }^{33}$ R. Casalini and S. Bair, The Journal of Chemical Physics 128, 084511 (2008).

${ }^{34}$ F. M. Gaciño, M. J. Comuñas, T. Regueira, J. J. Segovia, and J. Fernández, The Journal of Chemical Thermodynamics 87, 43 (2015).

${ }^{35}$ E. Thoms, A. Grzybowski, S. Pawlus, and M. Paluch, The Journal of Physical Chemistry Letters 9, 1783 (2018).

${ }^{36}$ C. Lederle, W. Hiller, C. Gainaru, and R. Böhmer, The Journal of Chemical Physics 134, 064512 (2011).

${ }^{37}$ S. Pawlus, S. Klotz, and M. Paluch, Physical Review Letters 110 (2013), 10.1103/physrevlett.110.173004.

${ }^{38}$ S. Pawlus, M. Paluch, and A. Grzybowski, The Journal of Chemical Physics 134, 041103 (2011).

${ }^{39}$ S. Bauer, K. Burlafinger, C. Gainaru, P. Lunkenheimer, W. Hiller, A. Loidl, and R. Böhmer, The Journal of Chemical Physics 138, 094505 (2013). 